\title{
La dieta y el orlistat ayudan a reducir el peso y la tensión arterial en pacientes hipertensos
}

\author{
Dietary changes and orlistat help in reducing blood pressure in hypertensive patients
}

\section{Objetivo}

Determinar los efectos sobre la tensión arterial (TA) en hipertensos esenciales, de las intervenciones dietarias, farmacológicas y quirúrgicas para reducir el peso corporal.

\section{Fuente y selección de datos}

MEDLINE, EMBASE, CINAHL y Cochrane, Agencia Europea para la Evaluación de Productos Medicinales (EMEA) y la Food and Drug Administration (FDA) de EE.UU., con una actualización de la búsqueda para incluir datos de rimonabant de 2007. Se incluyeron solamente ensayos controlados aleatorizados, realizados para comparar intervenciones dietarias, farmacológicas o invasivas para la pérdida de peso, con un seguimiento de más de 24 semanas. En caso de recibir otra terapia adicional (medicación antihipertensiva) ésta también

Tabla 1: efecto de dieta, la sibutramina y el orlistat sobre el peso y la tensión arterial en hipertensos esenciales.

\begin{tabular}{|c|c|c|c|c|}
\hline \multicolumn{2}{|c|}{ Variable } & Dieta vs. control & Orlistat vs. control & Sibutramina vs. control \\
\hline \multicolumn{2}{|l|}{ Peso } & $-4,14 \mathrm{~kg}(-4,98$ a $-3,30)$ & $-3,74 \mathrm{~kg}(-4,7$ a $-2,78)$ & $-3,72 \mathrm{~kg}(-4,85$ a $-2,59)$ \\
\hline \multirow{2}{*}{$\begin{array}{c}\text { Tensión } \\
\text { arterial }\end{array}$} & Sistólica & $-6,26 \mathrm{mmHg} \quad(-9,8$ a $-2,7)$ & $-2,46 \mathrm{mmHg}(-4,01$ a $-0,90)$ & No pudo realizarse meta-análisis ${ }^{2}$ \\
\hline & Diastólica & $-3,41 \mathrm{mmHg}(-5,55$ a $-1,27)$ & $-1,92 \mathrm{mmHg}(-2,99$ a $-0,85)$ & $+3,16 \mathrm{mmHg}(1,40$ a 4,92$)$ \\
\hline
\end{tabular}

${ }^{a}$ No pudo realizarse meta-análisis por falta de medidas de variabilidad de los estudios

\section{Conclusiones}

El tratamiento dietario y el uso de orlistat se asocian a una reducción significativa del peso corporal y de la TA en hipertensos. La sibutramina redujo el peso corporal pero aumentó la TA diastólica.

Palabras clave: descenso de peso, hipertensión arterial, orlistat, sibutramina, dieta. Key words: weight reduction, hypertension, orlistat, sybutramin, diet. Fuente de financiamiento: no reportada.

\section{Comentario}

Numerosas investigaciones epidemiológicas y clínicas asociaron la obesidad y la HTA, ambas formando parte del síndrome metabólico. Su tratamiento se centra en el descenso de peso, difícil de mantener en el largo plazo. Cuando se los adiciona a cambios en el estilo de vida, los fármacos autorizados actualmente para el tratamiento crónico de la obesidad -orlistat, sibutramina y rimonabant- promueven un descenso de peso adicional y lo más importante, una mejoría del perfil de riesgo cardiovascular, en relación al placebo. La sibutramina inhibe en forma selectiva la recaptación de serotonina, noradrenalina y dopamina, y ante la falta de estudios específicos, ha sido contraindicada en pacientes con riesgo cardiovascular elevado. Está en marcha el estudio SCOUT ${ }^{1}$-cuya finalización se estima para fines de 2008- aleatorizado y doble ciego, que pretende evaluar los efectos del tratamiento con sibutramina o placebo asociados a cambios en el estilo de vida en obesos con alto riesgo cardiovascular ${ }^{2}$. Según el informe publicado en 2007 a las seis semanas de iniciado, en los pacientes que redujeron más de un $5 \%$ de su peso corporal, la TA sistólica/diastólica disminuyó -7,5/-2,5 mmHg ( $p<0,001)$ en hipertensos y -2,0/0,25 ( $p<0,001$ y $p=0,591$, respectivamente) en normotensos. En cambio, quienes redujeron menos del $5 \%$ su peso, la sibutramina redujo la TA sistólica/diastólica en hipertensos -4,5/$1,5 \mathrm{mmHg}$, pero en normotensos se incrementó $1,5 \mathrm{mmHg}$ la sistólica y la diastólica $(p<0,001)$. Estos resultados iniciales y de corto plazo sugieren que los efectos de la sibutramina sobre la TA dependen de los valores previos de TA y del descenso de peso alcanzado.

También con posterioridad a la revisión que nos ocupa, se publicó un meta-análisis que mostró que rimonabant disminuye la TA en obesos hipertensos ${ }^{3}$.

\section{Conclusiones del comentador}

Además del tratamiento dietario y de la promoción del ejercicio, parece pertinente considerar la farmacoterapia en obesos hipertensos, teniendo en cuenta el limitado seguimiento de los estudios disponibles hasta la fecha.

Ana Mara Cappelletti y Marcos Mayer [ Universidad Favaloro, anacappelletti@yahoo.com.ar ]

Recibido 6/5/08 y aceptado el 07/6/08.

Cappelletti AM. La dieta y el orlistat ayudan a reducir el peso y la tensión arterial en pacientes hipertensos Evid. actual. práct. ambul; 11(4): 108, JulAgo.2008. Horvath K, Jeitler K, Siering U, Stich AK, Skipka G, Gratzer TW, et al. Long-term Effects of Weight-Reducing Interventions in Hypertensive Patients: Systematic Review and Meta-analysis. Arch Intern Med 2008;168(6):571-80. PMID: 18362248. Disponible en URL: http://archinte.ama-assn.org/cgi/reprint/168/6/571 (último acceso 04/08/08)..

Referencias

1.James WPT. The SCOUT study: risk-benefit profile of sibutramine in overweight high-risk cardiovascular patients. Eur Heart J Suppl 2005;7(suppl_L):L44-8.

2. Torp-Pedersen $\mathrm{C}$ y col. on the behalf of the SCOUT Investigators. Cardiovascular responses to weight management and sibutramine in high-risk subjects: an analysis from the SCOUT trial. European Heart Journal 2007;28:2915-2923.

3. Ruilopea $\mathrm{L}$ y col. Effect of rimonabant on blood pressure in overweight/obese patients with/without co-morbidities: analysis of pooled RIO study results. Journal of Hypertension $2008,26: 357-367$ 